\title{
On Some Problems with Nonlocal Integral Condition
}

\section{N. Sergejeva}

\author{
Department of Mathematics and Natural Sciences \\ Parades 1, LV-5400 Daugavpils, Latvia \\ E-mail(corresp.): natalijasergejeva@inbox.lv
}

Received August 30, 2009; revised January 4, 2010; published online February 15, 2010

\begin{abstract}
We study the second order nonlinear boundary value problems with nonlocal integral conditions and construct the Fučík type spectrum for these problems. Keywords: Fučík problem, Fučík spectrum, integral boundary value problem.
\end{abstract}

AMS Subject Classification: 34B15.

\section{Introduction}

The Fučík equation

$$
x^{\prime \prime}=-\mu x^{+}+\lambda x^{-}
$$

is a simple formally nonlinear equation with piece-wise linear right hand side. It was intensively investigated together with various boundary conditions ([6, $7,10]$ ), for instance, with the Dirichlet boundary conditions

$$
x(0)=0, \quad x(1)=0 .
$$

A set of $(\mu, \lambda)$ such that the problem has nontrivial solutions is called the Fučík spectrum. The problem (1.1), (1.2) generalizes classical spectral problems with one parameter and reduces to the classical problem if $\mu=\lambda$. The Fučík spectrum is a two-dimensional set which may have interesting properties. The Fučík spectrum for the problem (1.1), (1.2) is well known and consists of infinite set of curves (branches) which can be obtained analytically and graphically. The Fučík spectra for the Dirichlet and Neumann problems are similar, but the spectrum in the case of one (or two) of boundary conditions being in integral form differs essentially. It was studied in the work of the author ([11]) and both analytical and graphical description was given in the case of boundary conditions

$$
x(0)=0, \quad \int_{0}^{1} x(s) d s=0 .
$$


In this work we study more complicated case

$$
x(0)=0, \quad x(1)=\gamma \int_{0}^{1} x(s) d s, \quad \gamma \in \mathbb{R} .
$$

which includes both the conditions (1.2) and (1.3). We give description of the spectrum for various $\gamma$ and discover new properties of the spectrum.

The paper is organized as follows. In Section 2 we give results on the spectrum for the well known Fučík problem (1.1), (1.2). We give the basic notations also. In Section 3 we present the results on the spectrum for the Fučík type problem (1.1), (1.4). Our goal is to study the spectrum of the problem (1.1), (1.4). The properties of the spectrum for different values of $\gamma$ are given in Section 4. In Section 5 we consider more general problem and describe some properties of the spectrum. This study is based on some previous works. The first one is the author's work [11], where the spectrum of the equation (1.1) together with the integral condition (1.3) was considered and some properties of the spectrum were presented. Some branches of the spectrum for the problem (1.1), (1.4) consist of several components similarly to the spectrum for the problem $(1.1),(1.3)$. We also use papers $[3,8,9]$, where the eigenvalue problem for one-dimentional differential operator

$$
x^{\prime \prime}+\lambda x=0,
$$

together with integral conditions (1.4) was considered. Notice that the problem (1.1), (1.4) generalizes classical spectral problems with one parameter and reduces to the problem (1.5) if $\mu=\lambda$. We note that nonlocal boundary conditions (including integral conditions) are formulated for many applied problems, see e.g. [1, 2], where also numerical algorithms for solution of such problems are proposed and investigated.

\section{The Basic Statements}

Consider the classical Fučík problem (1.1), (1.2). First, we describe the decomposition of the spectrum into branches $F_{i}^{+}$and $F_{i}^{-}(i=0,1,2, \ldots)$.

Proposition 1. The Fučik spectrum consists of the set of the curves $F_{i}^{+}=$ $\left\{(\mu, \lambda) \mid x^{\prime}(0)>0\right\}$, the nontrivial solution of the problem (1.1), (1.2) $x(t)$ has exactly $i$ zeroes in $(0,1) ; F_{i}^{-}=\left\{(\mu, \lambda) \mid x^{\prime}(0)<0\right\}$, the nontrivial solution of the problem (1.1), (1.2) $x(t)$ has exactly $i$ zeroes in $(0,1)$.

Theorem 1. [5] The Fučik spectrum for the problem (1.1), (1.2) consists of the branches given by

$$
\begin{aligned}
& F_{0}^{+}=\left\{\left(\pi^{2}, \lambda\right)\right\}, \quad F_{0}^{-}=\left\{\left(\mu, \pi^{2}\right)\right\}, \\
& F_{2 i-1}^{+}=\left\{(\mu, \lambda) \mid i \frac{\pi}{\sqrt{\mu}}+i \frac{\pi}{\sqrt{\lambda}}=1\right\}, \quad F_{2 i}^{+}=\left\{(\mu, \lambda) \mid(i+1) \frac{\pi}{\sqrt{\mu}}+i \frac{\pi}{\sqrt{\lambda}}=1\right\}, \\
& F_{i}^{-}=\left\{(\mu, \lambda) \mid(\lambda, \mu) \in F_{i}^{+}\right\}, \quad i=1,2, \ldots
\end{aligned}
$$


The first five pairs of branches of the spectrum to the problem (1.1), (1.2) are depicted in Figure 1.

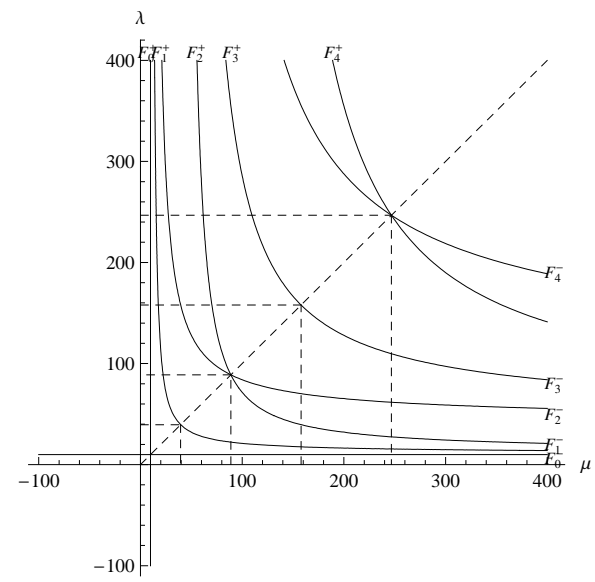

Figure 1. The spectrum for the problem (1.1), (1.2).

\section{The Spectrum of the Problem}

Now consider the problem (1.1), (1.4). The expressions for the branches of the spectrum for this problem are given in the next theorem. The meaning of the notation of the spectrum branches is the same as earlier.

Theorem 2. The spectrum $\sum_{\gamma}=\bigcup_{i=0}^{+\infty} F_{i}^{ \pm}$for the problem (1.1), (1.4) consists of the branches (if these branches exist for corresponding value of $\gamma$ ) given by (where $i=1,2, \ldots$ )

$$
\begin{gathered}
F_{0}^{+}=\left\{(\mu, \lambda) \mid \sinh \sqrt{-\mu}=\frac{\cosh \sqrt{-\mu}-1}{\sqrt{-\mu}} \gamma, \mu<0 ;\right. \\
\left.\mu=0 ; \sin \sqrt{\mu}=\frac{1-\cos \sqrt{\mu}}{\sqrt{\mu}} \gamma, 0<\mu \leq \pi^{2}\right\}, \\
F_{1}^{+}=F_{1+}^{+} \bigcup F_{1,0}^{+} \bigcup F_{1-}^{+}, \\
F_{1+}^{+}=\left\{(\mu, \lambda) \mid \frac{2}{\mu} \gamma-\frac{1}{\lambda} \gamma+\gamma \frac{\cos \left(\sqrt{\lambda}-\sqrt{\frac{\lambda}{\mu}} \pi\right)}{\lambda}+\frac{\sin \left(\sqrt{\lambda}-\sqrt{\frac{\lambda}{\mu}} \pi\right)}{\sqrt{\lambda}}=0,\right. \\
\left.\mu>\pi^{2}, \frac{\pi}{\sqrt{\mu}}+\frac{\pi}{\sqrt{\lambda}} \geq 1\right\},
\end{gathered}
$$




$$
\begin{aligned}
& F_{1,0}^{+}=\left\{(\mu, \lambda) \mid(\gamma-2) \mu+2(\pi-\pi \gamma) \sqrt{\mu}+\pi^{2} \gamma-4 \gamma=0,\right. \\
& \left.\mu>\pi^{2}, \lambda=0\right\} \\
& F_{1-}^{+}=\left\{(\mu, \lambda) \mid \frac{2}{\mu} \gamma-\frac{1}{\lambda} \gamma+\gamma \frac{\cosh \left(\sqrt{-\lambda}-\sqrt{-\frac{\lambda}{\mu}} \pi\right)}{\lambda}+\frac{\sinh \left(\sqrt{-\lambda}-\sqrt{-\frac{\lambda}{\mu}} \pi\right)}{\sqrt{-\lambda}}=0\right. \text {, } \\
& \left.\mu>\pi^{2}, \lambda<0\right\} \\
& F_{2 i}^{+}=\left\{(\mu, \lambda) \mid \frac{2 i+1}{\mu} \gamma-\frac{2 i}{\lambda} \gamma-\gamma \frac{\cos \left(\sqrt{\mu}-\sqrt{\frac{\mu}{\lambda}} \pi i+\pi i\right)}{\mu}\right. \\
& \left.-\frac{\sin \left(\sqrt{\mu}-\sqrt{\frac{\mu}{\lambda}} \pi i+\pi i\right)}{\sqrt{\mu}}=0, i \frac{\pi}{\sqrt{\mu}}+i \frac{\pi}{\sqrt{\lambda}}<1,(i+1) \frac{\pi}{\sqrt{\mu}}+i \frac{\pi}{\sqrt{\lambda}} \geq 1\right\}, \\
& F_{2 i+1}^{+}=\left\{(\mu, \lambda) \mid \frac{2 i+2}{\mu} \gamma-\frac{2 i+1}{\lambda} \gamma-\gamma \frac{\cos \left(\sqrt{\lambda}-\sqrt{\frac{\lambda}{\mu}} \pi(i+1)+\pi(i+1)\right)}{\lambda}\right. \\
& -\frac{\sin \left(\sqrt{\lambda}-\sqrt{\frac{\lambda}{\mu}} \pi(i+1)+\pi(i+1)\right)}{\sqrt{\lambda}}=0,(i+1) \frac{\pi}{\sqrt{\mu}}+i \frac{\pi}{\sqrt{\lambda}}<1, \\
& \left.(i+1) \frac{\pi}{\sqrt{\mu}}+(i+1) \frac{\pi}{\sqrt{\lambda}} \geq 1\right\}, \\
& F_{i}^{-}=\left\{(\mu, \lambda) \mid(\lambda, \mu) \in F_{i}^{+}\right\} .
\end{aligned}
$$

Proof. The proof of this theorem is similar to the proof given in the work [11] for the case of $(\mu, \lambda)$ being in the first quadrant. We will restrict to some comments. First of all, we obtain the expressions for $F_{0}^{+}$. Let's suppose that the solution without zeroes in the interval $(0,1)$ exists and $x^{\prime}(0)>0$. In this case we obtain that problem (1.1), (1.4) reduces to the eigenvalue problem. The solution of the problem must satisfy the condition (1.4), thus the expression for $F_{0}^{+}$for positive $\mu$ values is obtained. Analogously we do analysis for nonpositive $\mu$ values.

The idea of the proof for other branches is similar. We consider the eigenvalue problems in the intervals between two consecutive zeroes of the solution and use the conditions of the solutions for these problems. For example we will prove this theorem for $\mu>\pi^{2}, \lambda<0$ (this means that $(\mu, \lambda)$ are in the fourth quadrant). Suppose that $(\mu, \lambda) \in F_{1-}^{+}$and let $x(t)$ be the corresponding nontrivial solution of the problem (1.1), (1.4). The solution has only one zero in $(0,1)$ and $x^{\prime}(0)>0$. Let this zero be denoted by $\tau$. Consider a solution of the problem (1.1), (1.4) first in the interval $(0, \tau)$ and then in the interval $(\tau, 1)$. We obtain that the problem $(1.1),(1.4)$ in these intervals reduces to the linear eigenvalue problems. So in the interval $(0, \tau)$ we have the problem $x^{\prime \prime}=-\mu x$ with boundary conditions $x(0)=x(\tau)=0$. In the interval $(\tau, 1)$ we have the problem $x^{\prime \prime}=-\lambda x(\lambda<0)$ with boundary condition $x(\tau)=0$, notice that $x^{\prime}(\tau)<0$. Let the value of $-\lambda$ be denoted by $\delta$. 
In view of (1.4) a solution of the problem (1.1), (1.4) $x(t)$ must satisfy the condition

$$
x(1)=\gamma\left(\int_{0}^{\tau} x(s) d s+\int_{\tau}^{1} x(s) d s\right) .
$$

Since $x(t)=A \sin \sqrt{\mu} t$ for $A>0$ (this relation holds only for $t \in(0, \tau)$ ) we obtain that

$$
\tau=\frac{\pi}{\sqrt{\mu}}, \quad x^{\prime}\left(\frac{\pi}{\sqrt{\mu}}\right)=-\sqrt{\mu} A, \quad \int_{0}^{\tau} x(s) d s=\frac{2 A}{\sqrt{\mu}} .
$$

Now we consider a solution of the equation $x^{\prime \prime}=\delta x$ in $(\tau, 1)$. This solution is $x(t)=C_{1} \exp (\sqrt{\delta} t)+C_{2} \exp (-\sqrt{\delta} t)$. From $x^{\prime}\left(\frac{\pi}{\sqrt{\mu}}\right)=-A \sqrt{\mu}$, we obtain

$$
x(t)=-A \sqrt{\frac{\mu}{\delta}} \sinh \left(\sqrt{\delta t}-\sqrt{\frac{\delta}{\mu}} \pi\right), \quad x(1)=-A \sqrt{\frac{\mu}{\delta}} \sinh \left(\sqrt{\delta}-\sqrt{\frac{\delta}{\mu}} \pi\right) .
$$

The integral value is the following

$$
\int_{\tau}^{1} x(s) d s=A \frac{\sqrt{\mu}}{\delta}\left(1-\cosh \left(\sqrt{\delta}-\sqrt{\frac{\delta}{\mu}} \pi\right)\right) .
$$

From (3.1), (3.2), (3.3) we obtain

$$
-A \sqrt{\frac{\mu}{\delta}} \sinh \left(\sqrt{\delta}-\sqrt{\frac{\delta}{\mu}} \pi\right)=\gamma\left(\frac{2 A}{\sqrt{\mu}}+A \frac{\sqrt{\mu}}{\delta}\left(1-\cosh \left(\sqrt{\delta}-\sqrt{\frac{\delta}{\mu}} \pi\right)\right)\right) .
$$

Multiplying both sides by $1 /(A \sqrt{\mu})$ and replacing $\delta$ by $-\lambda$, we obtain the expression for $F_{1-}^{+}$.

\section{The Properties of the Spectrum}

Lemma 1. The branch $F_{0}^{ \pm}$of the spectrum for the problem (1.1), (1.4) exists only for $\gamma \geq 0$.

Proof. Let us consider $\gamma<0$. It is clear that $\operatorname{sign} \int_{0}^{1} x(s) d s \neq \operatorname{sign} x^{\prime}(0)$ for these values of $\gamma$. Therefore the branches $F_{0}^{ \pm}$do not exist for such $\gamma$ values. Now we consider $\gamma=0$. The spectrum for the problem (1.1), (1.4) is the classical Fučík spectrum with the branches $F_{0}^{ \pm}$. Let's consider the positive values of $\gamma$ and $x^{\prime}(0)>0$. Now we consider the expression for $F_{0}^{+}$for positive values of $\mu$

$$
\sin \sqrt{\mu}=\gamma(1-\cos \sqrt{\mu}) / \sqrt{\mu} .
$$

Rewrite the equation (4.1) in the form

$$
\sqrt{\mu} / \tan \frac{\sqrt{\mu}}{2}=\gamma
$$


Consider the left side of the equation (4.2) as a function. The range of values of this function is the interval $[0,2)$. Now we consider the expression for $F_{0}^{+}$ for negative values of $\mu$ and rewrite it in the form

$$
\sqrt{-\mu} / \tanh \frac{\sqrt{-\mu}}{2}=\gamma
$$

The studies of the equation (4.3) show that it is solvable only for $\gamma>2$. For $\gamma=2$ we obtain that $\mu=0$ is the branch $F_{0}^{+}$of the spectrum. Therefore the branch $F_{0}^{+}$exists only for $\gamma \geq 0$. The proof for $F_{0}^{-}$is similar.

Remark 1. The unique solution of the equation (4.1) where $0<\mu<\pi^{2}$ forms the straight line parallel to the $\lambda$ - axis (analogously the solution of the equation (4.3) for $\mu<0)$. This straight line is the branch $F_{0}^{+}$.

Lemma 2. The spectrum of the problem (1.1), (1.4) is not restricted to the first quadrant except for the value $\gamma=2$. For this value the spectrum is entirely in the first quadrant, the axes $\mu=0$ and $\lambda=0$ included.

Proof. It follows from Lemma 1 that for $0<\gamma<2$ the branches $F_{0}^{ \pm}$can be continued in the second and the fourth quadrants analogously as for the spectrum of the problem (1.1), (1.2) (see [5]), for $\gamma>2$ the branches $F_{0}^{ \pm}$are in the second, the third and the fourth quadrants, but for $\gamma=2$ the branches $F_{0}^{ \pm}$ are the axes $\mu=0$ and $\lambda=0$. For $\gamma<0$ and $\gamma>2$ the branches $F_{1}^{ \pm}$consist of three parts and form the continuous curves. These branches are located in the first, in the second and in the fourth quadrants. Let us consider the expression for $F_{1+}^{+}$

$$
\frac{2}{\mu} \gamma=\frac{1}{\lambda} \gamma-\gamma \frac{\cos \left(\sqrt{\lambda}-\sqrt{\frac{\lambda}{\mu}} \pi\right)}{\lambda}-\frac{\sin \left(\sqrt{\lambda}-\sqrt{\frac{\lambda}{\mu}} \pi\right)}{\sqrt{\lambda}} .
$$

Consider the right side of equation (4.4). Let $\lambda$ tends to 0. Using L'Hospital's rule, we obtain

$$
\lim _{\lambda \rightarrow 0} \frac{\cos \left(\sqrt{\lambda}-\sqrt{\frac{\lambda}{\mu}} \pi\right)-1}{\lambda} \gamma=-\frac{\gamma}{2}\left(1-\frac{\pi}{\sqrt{\mu}}\right)^{2} \lim _{\lambda \rightarrow 0} \frac{\sin \left(\sqrt{\lambda}\left(1-\frac{\pi}{\sqrt{\mu}}\right)\right)}{\sqrt{\lambda}\left(1-\frac{\pi}{\mu}\right)}=-\frac{\gamma}{2}\left(1-\frac{\pi}{\sqrt{\mu}}\right)^{2} .
$$

Now consider the last part of the equation (4.4). Let $\lambda$ tends to 0 .

$$
\lim _{\lambda \rightarrow 0} \frac{\sin (\sqrt{\lambda}-\sqrt{\lambda / \mu} \pi)}{\sqrt{\lambda}}=1-\frac{\pi}{\sqrt{\mu}} .
$$

It follows that for $\lambda$ tends to 0 the equation (4.4) tends to the next equation

$$
\frac{2}{\mu} \gamma-\frac{\gamma}{2}\left(1-\frac{\pi}{\sqrt{\mu}}\right)^{2}+1-\frac{\pi}{\sqrt{\mu}}=0
$$

Multiplying the equation (4.4) with $(-2 \mu)$, we obtain

$$
(\gamma-2) \mu+2(\pi-\pi \gamma) \sqrt{\mu}+\pi^{2} \gamma-4 \gamma=0
$$


or the expression for $F_{1,0}^{+}$. Using similar ideas we obtain the equation (4.5) for $F_{1-}^{+}$if $\lambda \rightarrow 0$. The investigation of (4.5) as quadratic equation for $k=\sqrt{\mu}$ with parameter $\gamma$ shows that solutions of this equation exist for any real $\gamma$ values, but these solutions satisfy the condition $\mu>\pi^{2}$ only for $\gamma<0$ and $\gamma>2$. This proves the lemma.

Lemma 3. There are the points of $(\mu, \lambda)$ - plane, which are fixed for any values of $\gamma$. For any values of $\gamma$, except $\gamma=0$, the odd-numbered and the evennumbered branches are separated by the $\left((2 i \pi)^{2},(2 i \pi)^{2}\right)$, but the even-numbered and the odd-numbered branches are separated by the points

$$
\begin{aligned}
& F_{2 i}^{+} \cap F_{2 i+1}^{+}=\left((\sqrt{i(i+1)}+i)^{2} \pi^{2} ;(\sqrt{i(i+1)}+i+1)^{2} \pi^{2}\right), \\
& \left.F_{2 i}^{-} \cap F_{2 i+1}^{-}=(\sqrt{i(i+1)}+i+1)^{2} \pi^{2},(\sqrt{i(i+1)}+i)^{2} \pi^{2}\right),
\end{aligned}
$$

where $i=1,2, \ldots$

Proof. It is clear that the odd-numbered branches and the even-numbered ones (and vice versa also) intersect at the points in which the problem (1.1), (1.4) reduces to the problem $(1.1),(1.3)$. It follows that these points are the same as for the problem $(1.1),(1.3)$ (see [11]).

Remark 2. It was observed in $[3,9]$ that some points of the spectrum for problems (1.5), (1.4) are fixed for any value of $\gamma$. These points were called constant eigenvalues points. The similar fixed points exist both on and aside the bisectix for the problem (1.1), (1.4) (they are depicted by large points in Figures 2, 3, 4).

Lemma 4. The spectrum of the problem (1.1), (1.4) for $\gamma \rightarrow 0$ is similar to the classical Fučik spectrum, but all pairs of the spectrum branches do not coincide. The spectrum of the problem (1.1), (1.4) for $\gamma \rightarrow \pm \infty$ tends to the spectrum of the problem (1.1), (1.3).

First let $\gamma \rightarrow 0$. Consider the second condition of (1.4). We obtain

$$
x(1)=\lim _{\gamma \rightarrow 0} \gamma \int_{0}^{1} x(s) d s=0
$$

or the conditions (1.2) for the classical Fučík problem.

Proof. Now let us consider $\gamma \rightarrow \pm \infty$. We rewrite the integral condition of the problem (1.1), (1.4) in the form

$$
\frac{x(1)}{\gamma}=\int_{0}^{1} x(s) d s,
$$

we can do it because $\gamma \neq 0$. The left side tends to zero and the integral condition tends to that in (1.3). 


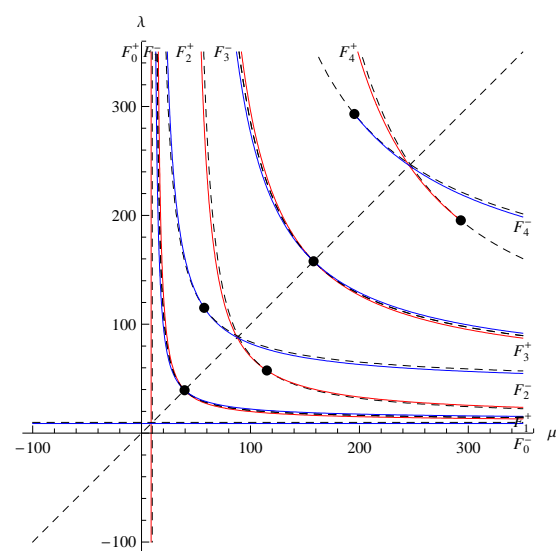

$\gamma=0.25$

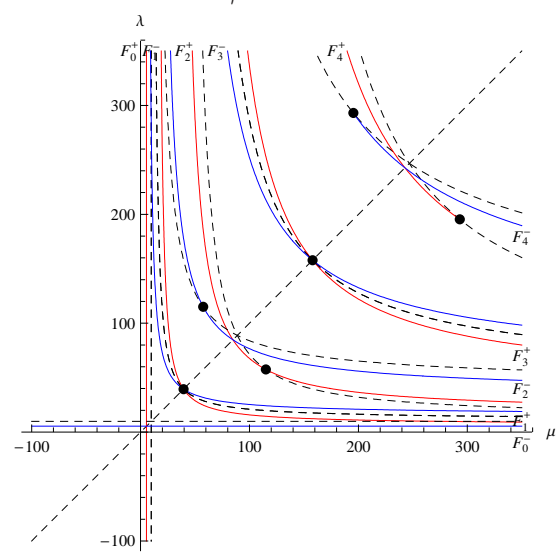

$\gamma=1$

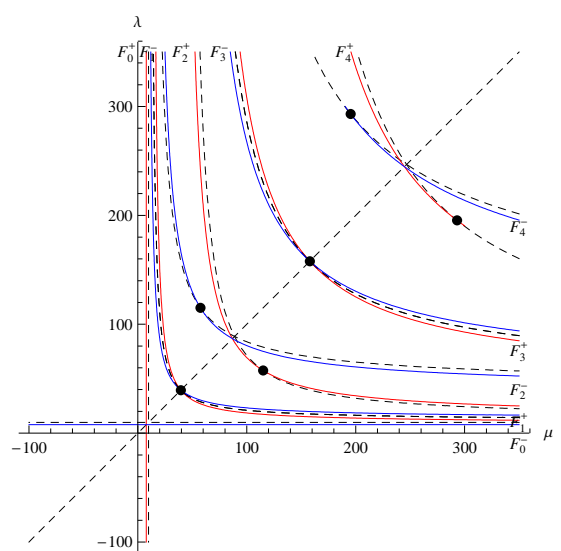

$\gamma=0.5$

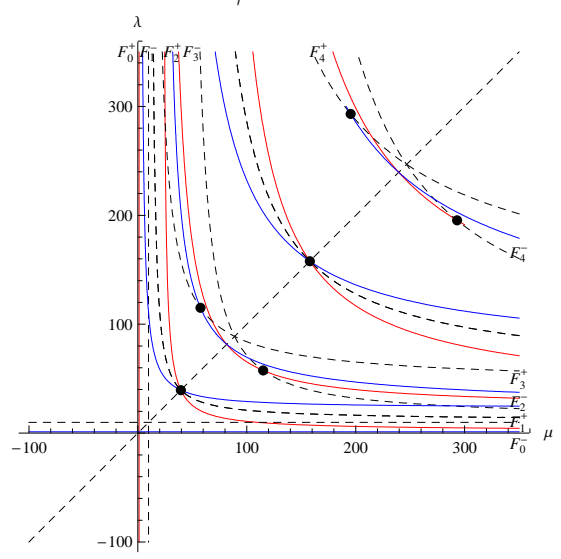

$\gamma=1.8$

Figure 2. The spectrum for the problem (1.1), (1.4) for different $\gamma$ values.

Next two theorems are direct consequences of Lemmas.

Theorem 3. The following properties of the spectrum for the problem (1.1), (1.4) for $0 \leq \gamma<2$ hold:

1. The branches $F_{0}^{ \pm}$of the spectrum for the problem (1.1), (1.4) exist.

2. All the branches of the spectrum are in the first quadrant (except $F_{0}^{ \pm}$, which are the straight lines and may be continued in the second and fourth quadrants).

3. The spectrum of the problem (1.1), (1.4) for $\gamma=0$ is the spectrum of the problem (1.1), (1.2). The odd branches of the spectrum $\left(F_{1}^{+}\right.$and $F_{1}^{-}, F_{3}^{+}$ and $F_{3}^{-}$etc.) coincide, the even ones don't coincide.

4. The spectrum of the problem (1.1), (1.4) is symmetric to the bisectrix of the first and the third quadrants; 

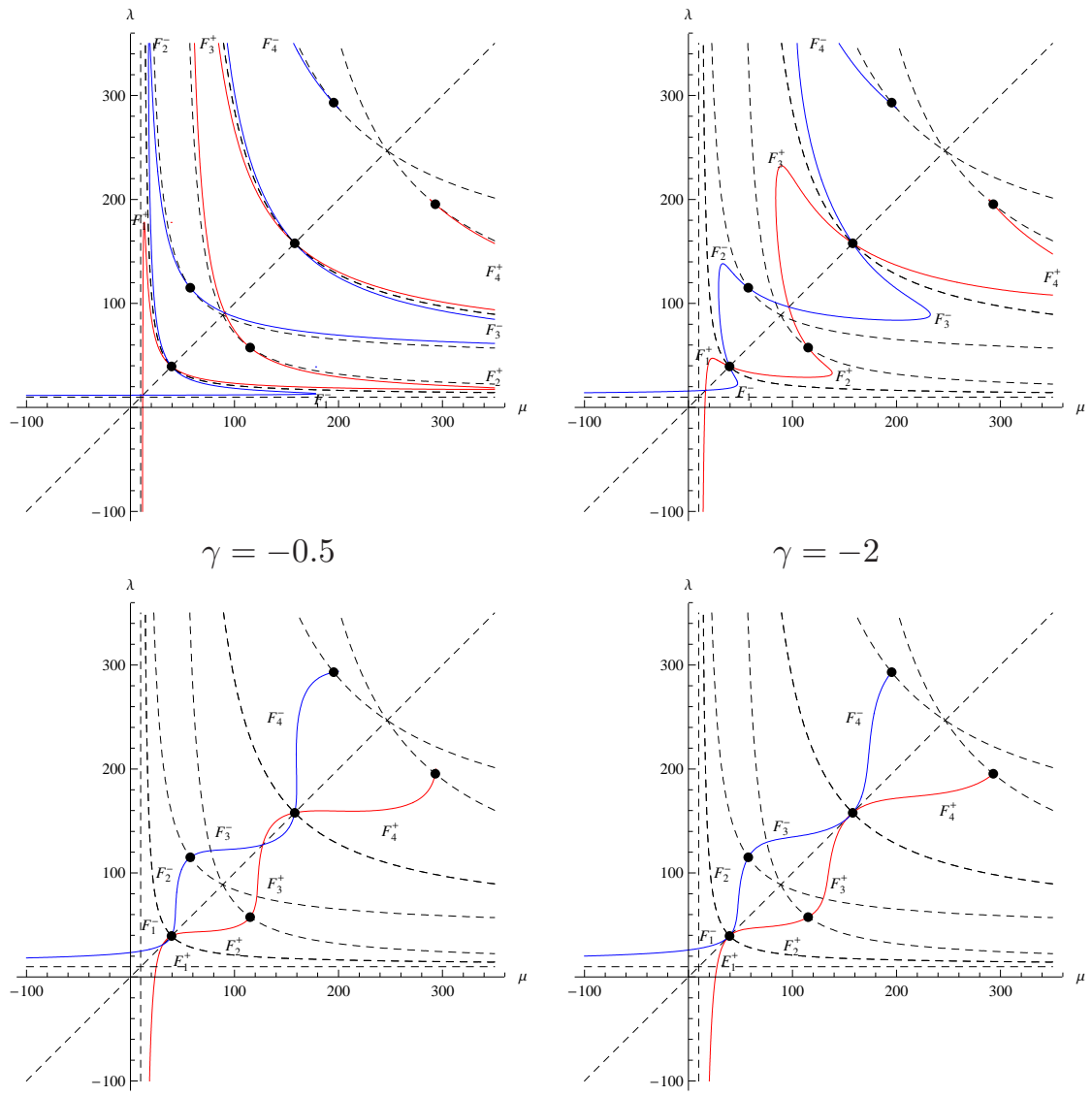

$\gamma=-15$

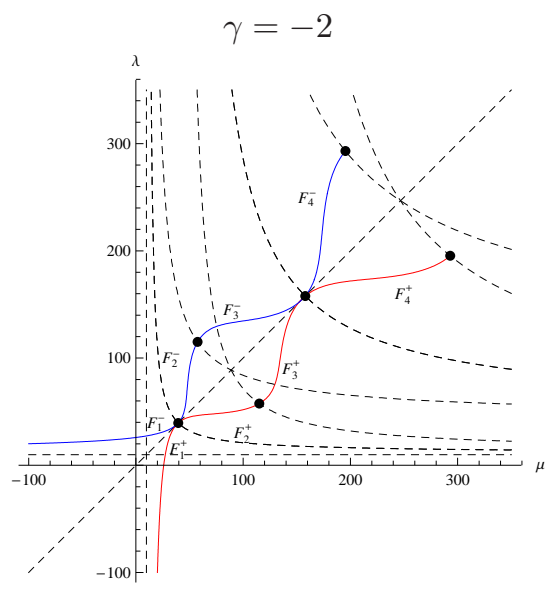

$\gamma=-100$

Figure 3. The spectrum for the problem (1.1), (1.4) for different $\gamma$ values.

5. The spectrum of the problem (1.1), (1.4) for $\gamma \rightarrow 0$ is similar to the spectrum of the problem (1.1), (1.2), but all pairs of branches of the spectrum do not coincide (in contrast to the case of classical Fučik spectrum).

Several first branches of the spectrum to the problem (1.1), (1.4) for $0 \leq \gamma<$ 2 are depicted in Figure 2, the dashed curves is the spectrum for the problem (1.1), (1.2), the red ones $-F_{i}^{+}$branches, the blue curves - $F_{i}^{-}$branches of the spectrum for the problem (1.1), (1.4).

Theorem 4. The following properties of the spectrum for the problem (1.1), (1.4) for $\gamma \in(-\infty, 0) \cup[2 ;+\infty)$ hold:

1. The branches $F_{0}^{ \pm}$of the spectrum for the problem (1.1), (1.4) exist only for $\gamma \in[2 ;+\infty)$.

2. The branch $F_{0}^{+}$of the spectrum for the problem (1.1), (1.4) (for $\gamma \in$ 
$[2 ;+\infty))$ is the asymptote for the branch $F_{1}^{-}$, the branch $F_{0}^{-}$is the asymptote for the branch $F_{1}^{+}$.

3. The union of the positive branches $\bigcup_{i=1}^{+\infty} F_{i}^{+}$and the negative ones $\bigcup_{i=1}^{+\infty} F_{i}^{-}$ form the continuous curves.

4. The branches of the spectrum are located in all the $(\mu, \lambda)$ plane, except $\gamma=2$. For $\gamma=2$ the spectrum is located in the first quadrant included the axes.

5. The spectrum of the problem (1.1), (1.4) is symmetric with respect to the bisectrix;

6. The spectrum of the problem (1.1), (1.4) for $\gamma \rightarrow \pm \infty$ tends to the spectrum of the problem (1.1), (1.3).

Several first branches of the spectrum to the problem (1.1), (1.4) for different $\gamma \in(-\infty, 0) \cup[2 ;+\infty)$ are depicted in Figures 3, 4. As earlier the dashed curves are the spectrum for the problem (1.1), (1.2), the red ones $-F_{i}^{+}$branches, the blue curves $-F_{i}^{-}$branches of the spectrum for the problem (1.1), (1.4).

Remark 3. The spectrum of the problem (1.1), (1.4) for $\gamma=2$ is in the first quadrant and the axes also belong the spectrum. For $\gamma=20$ and $\gamma=100$ the branches $F_{0}^{ \pm}$exist also, but they are far away from the axes (see Figure 4).

Remark 4. In the work [11] the Fučik equation was considered together with the conditions

$$
x(0)=0, \quad(1-\alpha) x(1)+\alpha \int_{0}^{1} x(s) d s=0, \quad \alpha \in[0,1]
$$

and some properties of the spectrum were described. The problem (1.1), (4.6) is a particular case of the problem (1.1), (1.4). Indeed, rewrite the second condition of the (4.6) (for $\alpha \neq 1$ ) in the form

$$
x(1)=\frac{\alpha}{\alpha-1} \int_{0}^{1} x(s) d s .
$$

Denote $\frac{\alpha}{\alpha-1}$ by $\gamma$. It follows that $\gamma \in(-\infty, 0]$. 

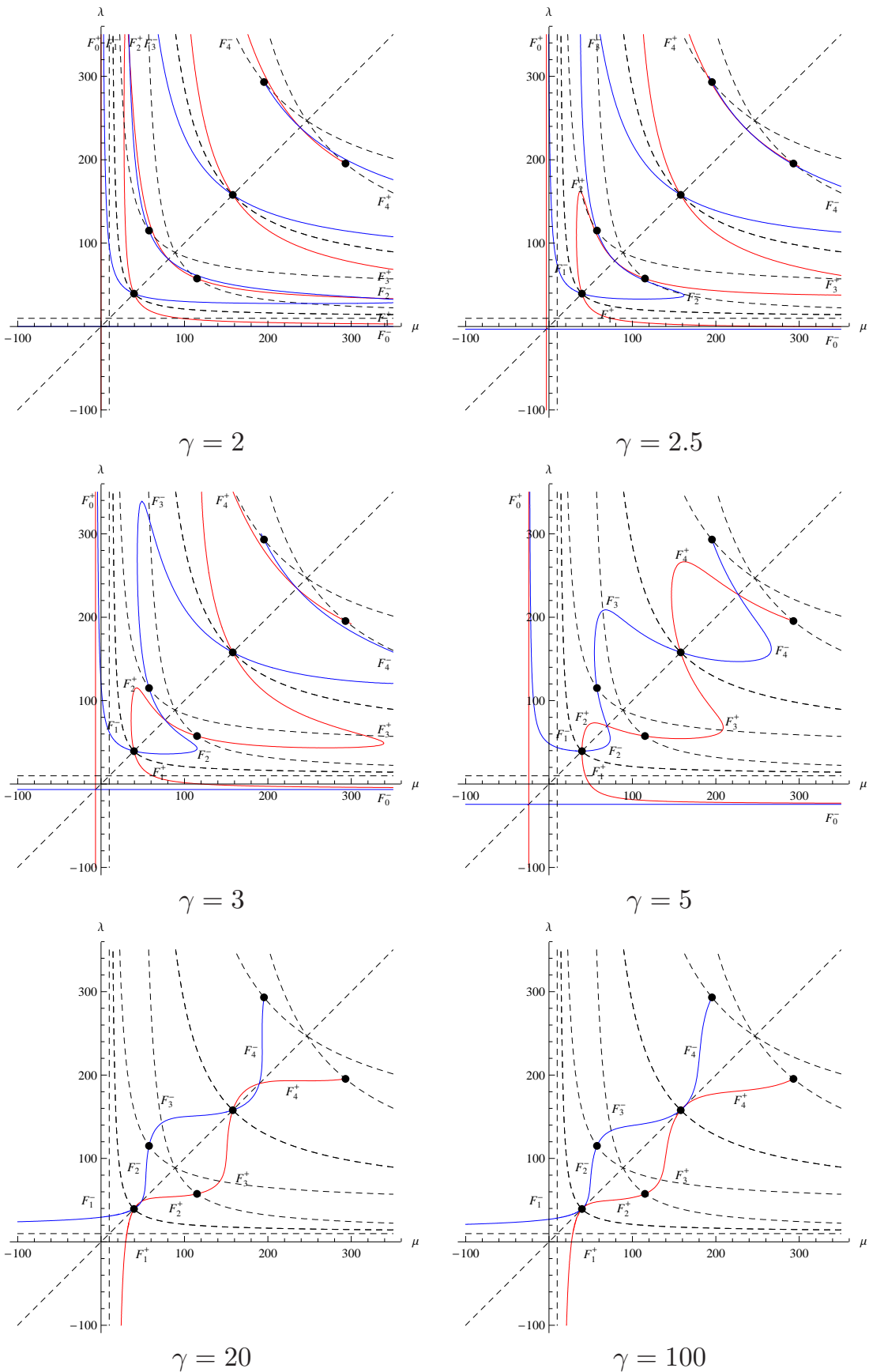

Figure 4. The spectrum for the problem (1.1), (1.4) for different $\gamma$ values. 


\section{More General Problem}

Now we consider the Fučík equation

$$
-x^{\prime \prime}=\mu x^{+}-\lambda x^{-},
$$

together with nonlocal boundary conditions

$$
x(0)=\gamma_{1} \int_{0}^{1} x(s) d s, \quad x(1)=\gamma_{2} \int_{0}^{1} x(s) d s, \quad \gamma_{1}, \gamma_{2} \in \mathbb{R} .
$$

The analog of this problem for one-dimensional differential operator was considered in $[3,8,9]$.

Lemma 5. The branch $F_{0}^{ \pm}$of the spectrum for the problem (5.1), (5.2) exists only for $\gamma_{1}+\gamma_{2} \geq 0$.

Proof. We consider the solutions of the problem (5.1), (5.2) without zeroes in the interval $(0 ; 1)\left((\mu, \lambda) \in F_{0}^{ \pm}\right)$. Let's consider the case $x(t)>0$. We obtain that the problem reduces to the eigenvalue problem. It is clear that $\mu \in\left(-\infty, \pi^{2}\right)$. The solution of the problem (5.1), (5.2) for $\mu \in\left(0, \pi^{2}\right)$ is

$$
x(t)=A \sin (\sqrt{\mu} t+\varphi)=A \sin (\sqrt{\mu}(t+\tau)),
$$

where $0 \leq \tau \leq \frac{\pi}{\sqrt{\mu}}-1$ ). It follows from the boundary conditions that

$$
\left\{\begin{array}{l}
\sqrt{\mu} \sin \sqrt{\mu} \tau=2 \gamma_{1} \sin \frac{\sqrt{\mu}}{2} \sin \left(\frac{\sqrt{\mu}}{2}+\sqrt{\mu} \tau\right), \\
\sqrt{\mu} \sin (\sqrt{\mu}+\sqrt{\mu} \tau)=2 \gamma_{2} \sin \frac{\sqrt{\mu}}{2} \sin \left(\frac{\sqrt{\mu}}{2}+\sqrt{\mu} \tau\right) .
\end{array}\right.
$$

The last system of equations yields

$$
\sqrt{\mu} / \tan \frac{\sqrt{\mu}}{2}=\gamma_{1}+\gamma_{2}
$$

Similarly as for the equation (4.1) the equation (5.3) has the solutions only for $0 \leq \gamma_{1}+\gamma_{2}<2$.

The solution of the problem (5.1), (5.2) for $\mu=0$ is $x(t)=A+B t$. We obtain from the boundary conditions that if $\gamma_{1}+\gamma_{2}=2$ then $\mu=0$ is an eigenvalue the corresponding eigenvalue problem.

The solution of the problem (5.1), (5.2) for $\mu<0$ is $x(t)=A \sinh (\sqrt{-\mu} t+$ $\varphi)=A \sinh (\sqrt{-\mu}(t+\tau))$ (where $\tau \geq 0)$. It follows from the boundary conditions that

$$
\sqrt{-\mu} / \tanh \frac{\sqrt{-\mu}}{2}=\gamma_{1}+\gamma_{2}
$$

Similarly as for the equation (4.3) the equation (5.4) has the solutions only for $\gamma_{1}+\gamma_{2}>2$.

Lemma 6. The spectrum of the problem (5.1), (5.2) for $\gamma_{1} \rightarrow 0$ and $\gamma_{2} \rightarrow 0$ tends to the spectrum of the classical Fučík problem (1.1), (1.2). 
Proof. Consider $\gamma_{1} \rightarrow 0$ and $\gamma_{2} \rightarrow 0$. It is clear that the conditions (5.2) tend to the conditions of the problem (1.1), (1.2).

Lemma 7. The spectrum of the problem (5.1), (5.2) for $\gamma_{1}=a$ (a-fixed number) and $\gamma_{2} \rightarrow \pm \infty$ tends to the spectrum of the problem (1.1), (1.3).

Proof. Consider $\gamma_{2} \rightarrow \pm \infty$. Let us write the second integral condition of the problem (5.1), (5.2) in the form $x(1) / \gamma_{2}=\int_{0}^{1} x(s) d s$. It follows that

$$
\int_{0}^{1} x(s) d s=0 .
$$

Therefore the first condition of the problem (5.1), (5.2) reduces to the

$$
x(0)=\gamma_{1} \int_{0}^{1} x(s) d s=0 .
$$

It is clear that the conditions (5.6), (5.5) are the conditions of the problem (1.1), (1.3).

Lemma 8. The spectrum of the problem (5.1), (5.2) for $\gamma_{1} \rightarrow \pm \infty$ and $\gamma_{2}=b$ ( $b$ is a fixed number) then the spectrum tends to the spectrum of the problem

$$
\begin{aligned}
& -x^{\prime \prime}=\mu x^{+}-\lambda x^{-}, \\
& x(1)=0, \quad \int_{0}^{1} x(s) d s=0 .
\end{aligned}
$$

The proof is similar to the proof of Lemma (7).

Remark 5. The spectrum of the problem (5.7), (5.8) coincides with the spectrum of the problem (1.1), (1.3).

The proof of this fact is clear. Let us change the variable in the problem (5.7), (5.8) as follows $X(\tau)=x(1-t)$. Then we obtain

$$
\begin{aligned}
& X^{\prime}=\frac{d X(\tau)}{d \tau}=\frac{d x(1-\tau)}{d \tau}=-\frac{d x(1-\tau)}{d(1-\tau)}=-\frac{d x(t)}{d t}=-x^{\prime}, \\
& X^{\prime \prime}=\frac{d^{2} X}{d \tau^{2}}=\frac{d}{d \tau} \frac{d X(\tau)}{d \tau}=-\frac{d}{d \tau} \frac{d x(t)}{d t}=-\frac{d}{d t} \frac{d x(t)}{d t} \frac{d t}{d \tau}=\frac{d^{2} x(t)}{d t^{2}}=x^{\prime \prime}
\end{aligned}
$$

or we have

$$
\begin{aligned}
& -X^{\prime \prime}=\mu X^{+}-\lambda X^{-} \\
& X(0)=x(1)=0, \quad \int_{0}^{1} X(s) d s=0 .
\end{aligned}
$$

Acknowledgement. The author wishes to thank the anonymous referee for the constructive suggestions. 


\section{References}

[1] R. Ciegis. Economical difference schemes for the solution of a two dimensional parabolic problem with an integral condition. Differential Equations, 41(3):10251029, 2005.

[2] R. Čiegis. Parallel numerical algorithms for $3 \mathrm{~d}$ parabolic problem with nonlocal boundary condition. Informatica, 17(3), 2006.

[3] R. Čiupaila, Ž. Jesevičiūtè and M. Sapagovas. On the eigenvalue problem for the one dimensional diffrerential operator with nonlocal integral condition. Nonlinear Analysis: Modelling and Control, 9(2):109-116, 2004.

[4] A. Gritsans, F. Sadyrbaev and N. Sergejeva. Two-parameter nonlinear eigenvalue problems. In A. Cabada, E. Liz and J.J. Nieto (Eds.)(Eds.), Proc. of the International Conference on Boundary Value Problems, Spain, 2008, volume 1124, pp. 185-194, 2009. AIP

[5] A. Kufner and S. Fučík. Nonlinear Differential Equations. Nauka, Moscow, 1988. (in Russian)

[6] A.C. Lazer and P.J. McKenna. Large-amplitude periodic oscillations in suspension bridges: Some new connections with nonlinear analysis. SIAM Review, 32(4):537-578, 1990. Doi:10.1137/1032120.

[7] P. Nečesal. Nonlinear boundary value problems with asymetric nonlinearities periodic solutions and the Fučik spectrum. University of West Bohemia, Czech Republic, 2003. PhD thesis

[8] S. Pečiulytė, O. Štikonienè and A. Štikonas. Sturm-Liouville problem for stationary differential operator with nonlocal integral boundary condition. Math. Model. Anal., 10(4):377-392, 2005.

[9] S. Pečiulytė, O. Štikonienè and A. Štikonas. Investigation of negative critical points of the characteristic function for problems with nonlocal boundary conditions. Nonlinear Anal. Model. Control, 13(4):467-490, 2008.

[10] B.P. Rynne. The Fučík spectrum of general Sturm-Liouville problems. Journal of Differential Equations, 161(1):87-109, 2000. Doi:10.1006/jdeq.1999.3661.

[11] N. Sergejeva. On the unusual Fučík spectrum. In B. Belinsky, K. Lan, X. Lu, M. Miranville and R. Shivaji(Eds.), Proc. for the 6th AIMS International Conference on DCDE, France, 2006, On the unusual Fučík spectrum, pp. 920-926. AIMS, 2007. 